\title{
Sustainment and Additional Heating of High-Beta Field-Reversed Configuration Plasmas
}

\author{
S. Okada, T. Fukuda, K. Kitano, H. Sumikura, T. Higashikozono, M. Inomoto, S. Yoshimura, \\ M. Ohta and S. Goto \\ Science and Technology Center for Atoms, Molecules and Ions Control, Osaka University. \\ Yamada-oka 2-1, Suita city, Osaka, Japan \\ E-mail contact of main author: okada@ppl.eng.osaka-u.ac.jp
}

\begin{abstract}
In additional heating experiment of translated theta-pinch produced Field-Reversed-Configuration (FRC) plasma with density of $5 \times 10^{19} / \mathrm{m}^{3}$ and pressure balance temperature of $100 \mathrm{eV}$ by $10 \mathrm{keV}$ hydrogen neutral beam, only electron is observed to be heated; electron and ion temperature is controlled independently for the first time in the FRC plasma. So far, confinement times of the FRC plasma were known to depend approximately on $R^{2} / \rho_{i o}$ ( $R$; major radius, $\rho_{i o}$; ion gyro radius) and dependence on electron temperature was small, which is confirmed by the present experiment. The FRC plasma is also produced and sustained for longer than $4 m s$ by a rotating magnetic field (RMF) in a metal confinement chamber. Though the performance of the present RMF produced FRC plasma is low, or the density and the temperature are lower than $5 \times 10^{17} / \mathrm{m}^{3}$ and $10 \mathrm{eV}$ respectively, and can be sustained in quartz chamber. But future high temperature FRC plasma should be sustained in metal chamber. The efficiency of the FRC plasma production is higher for higher RMF frequency.
\end{abstract}

\section{Introduction}

A plasma with field reversed configuration (FRC) [1] consists only of ploidal field and does not have toroidal field. From this fact, plasma beta value $\beta$ or the plasma pressure normalized by the external magnetic pressure is $100 \%$ at the magnetic axis $r=R$ and even the beta value averaged inside the separatrix $r=r_{s}$ takes the value of as large as about $90 \%$. The strength of the magnetic field changes from its maximum value of $B_{w}$ at just inside a metal wall of a confinement vessel $\left(r=r_{w}\right)$ to zero at $r=R$ and further, to negative. Behavior of slow wave is studied from practical interest in heating high beta plasma and also from physical interest in wave propagation in the plasma with strong spatial variation in magnetic field [2] [3]. Extreme high beta nature, together with the fact that the FRC plasma is produced in an apparatus with linear configuration, is also attractive from reactor point of view [4]. But the FRC plasma, which has thus far the best performance (largest triple product $n \tau T)$ - a theta pinch produced FRC plasma, can not be sustained by the most economical Ohmic heating, because no material structure is linking the plasma. In order to seek for the possibility to additionally heat and/or sustain the FRC plasma, neutral beam (NB) injection experiment was carried out on the FRC Injection Experiment (FIX) apparatus in Osaka University [5] [6]. In the experiment, NB was injected into the plasma which was confined in the magnetic configuration with stronger mirror field than normal operating condition. In the case, confinement times were shorter (degraded) than normal operating condition without NB injection. But with NB the confinement times more than recovered and surpassed those of the normal operating condition. In this work, NB injection experiment was done on the plasma with stronger mirror field as before but without degraded confinement. Confinement times elongated with NB injection as before and detailed measurement was carried out to interpret the result.

For the FRC reactor, it is not enough to sustain the theta-pinch-produced FRC plasma but must drive toroidal current and increase confinement field or increase trapped flux [7]. For this purpose, utilization of rotating magnetic field (RMF) is proposed [7] and the experiment 
is carried out in Washington University [8]. As the frequency $f$ of the RMF must exceed the ion gyro frequency $f_{c i}$ of the RMF magnetic field strength, the RMF is applied from outside of the quartz discharge tube. At a present stage, the plasma temperature is $10-100 \mathrm{eV}$. But in future, plasma temperature should be higher and metal confinement chamber will be required. In this work, study on production and sustainment of the FRC plasma by RMF in a metal chamber is shown.

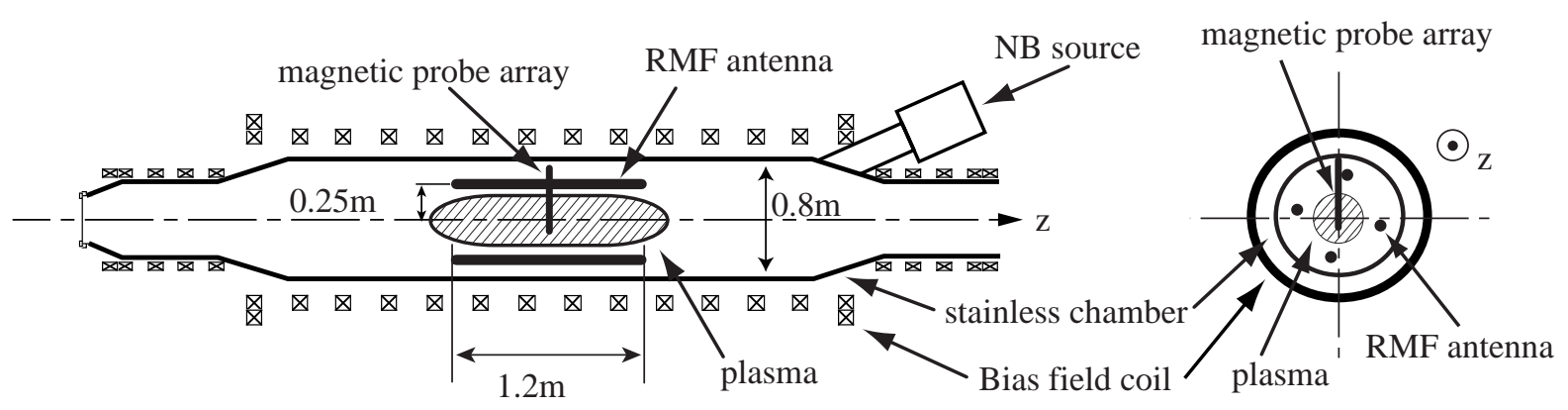

FIG.1 Schematic layout of FIX apparatus with RMF and NB systems.

\section{Experimental Arrangement}

The FRC plasma is produced in a $0.3 m$-diameter, $1.16 m$-long negatively biased theta pinch and is translated into a $0.8 m$-diameter, $3.4 m$-long metal confinement chamber. As the rise time of the NB is longer than the life time or duration of the plasma, the NB is introduced in advance before the plasma is introduced into the chamber. The angle between the axis of the NB injector and that of the confinement chamber is chosen to be $19.3 \mathrm{deg}$. with the intention to increase beam energy (Wperp +Wpara) and thus to draw much NB current keeping $W p e r p$ in such a way that the gyro-radius of ionized beam does not exceed $r_{w}$. In the axial direction, ionized beam is trapped between mirror fields installed at both ends of the confinement chamber with the mirror ratio of 8 to 14 .

In the case of the production and sustainment experiment of the FRC plasma by RMF, $12 m$-long, 4 -antenna rods were arranged at $r=0.25 m$ in the central part of the confinement chamber. Each rod was sheathed with insulator and fed from inverter switching circuit through air core transformers. Insulated Gate Bipolar Transistors (IGBT) were used in the inverter. By exciting each rod successively with phase difference of $\pi / 2$, rotating magnetic field $B_{R M F}$ of $0.1-3 m T$ were obtained at RMF frequency $f_{R M F}$ of $60-160 \mathrm{kHz}$. Arrangement for NB injection experiment and RMF experiment are shown in Fig.1.

\section{Additional heating experiment}

As the separatrix shape of the FRC plasma is extremely elongated axially, the separatrix radius $r_{s}$ at each axial position is obtained from a diamagnetic probe at each location with considerable accuracy. When the plasma is heated, as the plasma length is limited by a pair of mirror fields at both ends of the confinement chamber, the plasma expands radially. Then, as the plasma is in a flux conserver (metal confinement chamber), magnetic field strength between the separatrix and the metal wall increases. The radial expansion ceases when the plasma pressure and the external magnetic pressure balances. Obviously, plasma density should decrease with heating because of the radial expansion of the plasma. It must be noted that the number of particle supplied from the $\mathrm{NB}$ is order of $10^{-3}$ of the total particle 
inventory. In Fig.2, temporal development of (a) $r_{s}$, (b) separatrix volume obtained from axial array of magnetic probes, (c) chord averaged electron density inferred from side-on $\mathrm{CO}_{2}$ interferometer and $r_{s}$, (d) pressure balance temperature $T_{t o t}$ are shown for the cases without NB and with hydrogen NB $(10 \mathrm{keV}, 177 \mathrm{~kW})$ into deuterium plasma. Plasma heating and increase of $r_{s}$ and decrease of electron density by the NB injection is apparently observed. In Fig.3, results of similar experiments carried out with $10 \mathrm{keV}, 170 \mathrm{~kW}$ helium-NB are shown. In this case, heating and associated phenomena are not observed. In Fig.4, dependence of confinement times on injected NB power is shown. Energy loss time $\tau_{E}$ defined by $\tau_{E}=1 /\left(\left(d E_{t o t} / d t\right) / E_{t o t}\right)$ in which $E_{t o t}$ is the total (plasma + magnetic) energy of the FRC plasma, decay time $\tau_{\phi}$ of trapped magnetic flux and particle confinement time $\tau_{N}$ are shown by green, blue and red color, respectively. Injected NB power was 137 , 177 and $251 \mathrm{~kW}$. In this case, fraction of the trapping of ionized NB becomes by far smaller than other cases and $D_{\alpha}$ increases, signifying deleterious effect has been caused by inappropriately high energy NB. Dependence of $\tau_{\phi}$ on $T_{e}$ by Thomson scattering measured at the axial midplane at $r=5 \mathrm{~cm}$ is shown in Fig.5. Dotted line signifies $T_{e}^{3 / 2}$ dependence. At a glance, $\tau_{\phi}$ seems to depend on $T_{e}$. But, when $\tau_{\phi} / r_{s}{ }^{2}$ is plotted against $T_{e}$, having in mind the empirical scaling law that confinement times of the FRC plasma depends approximately on $r_{s}^{2} / \rho_{i o}, T_{e}$ dependence is not seen (Fig.6). In Fig.7, $\tau_{N} / \tau_{\phi}$ and $\tau_{E} / \tau_{\phi}$ is shown for different NB power. It is seen that those ratios do not depend on NB power. These results signify that confinement times elongate with NB injection and the elongation can be explained by the empirical scaling law; the value of $r_{s}$ increases with the plasma heating by NB injection, and it causes elongation in confinement times. In Fig.8, $T_{e}$ by Thomson scattering and ion temperature $T_{i}$ by OV Doppler broadening are shown for the case with and without NB. Plasma heating is seen to be ascribed to electron heating.
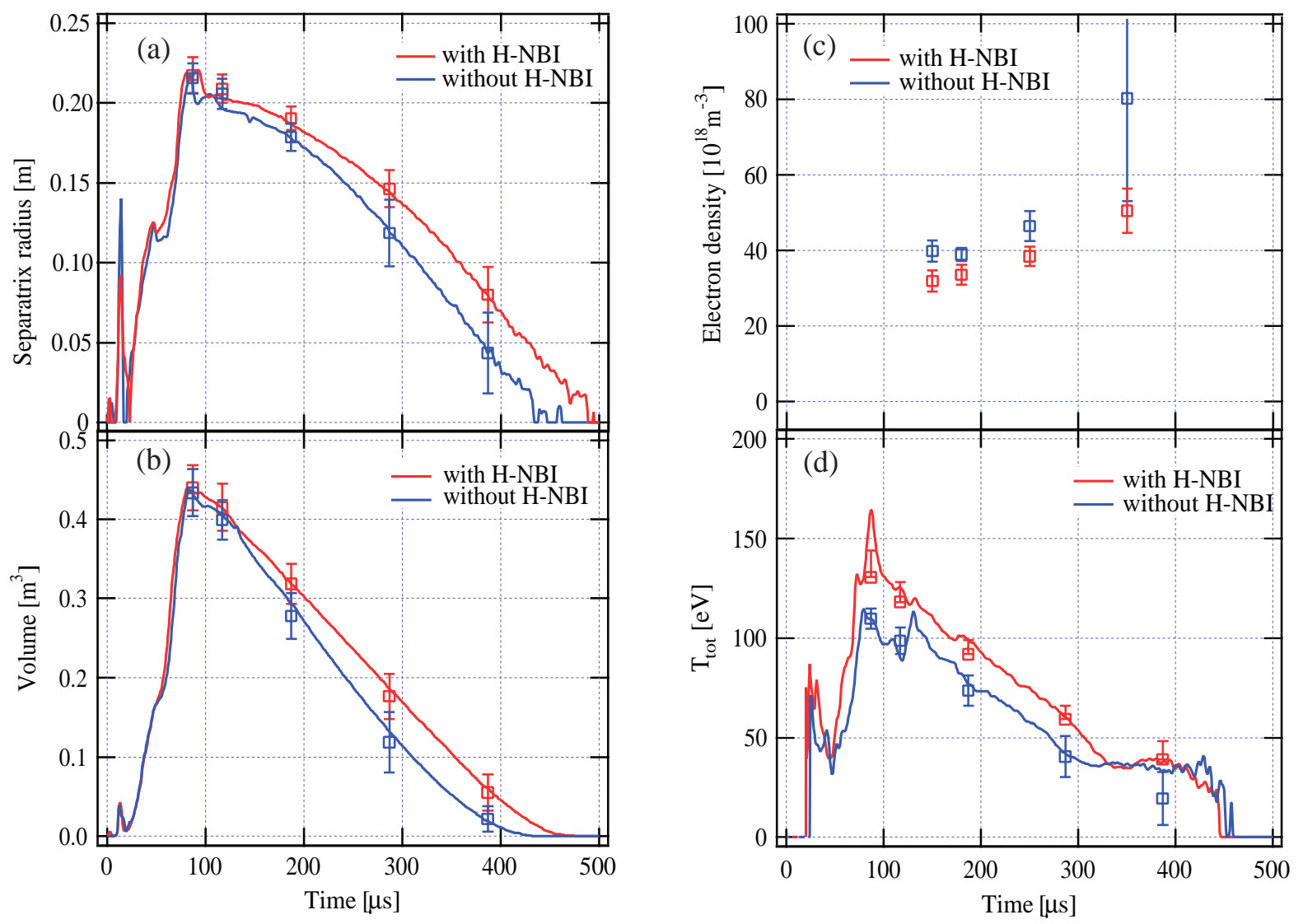

FIG.2 Temporal development of (a) $r_{s}$, (b) separatrix volume, (c) chord averaged electron density, (d) temperature $T_{\text {tot }}$ without $N B$ and with hydrogen $N B$. 

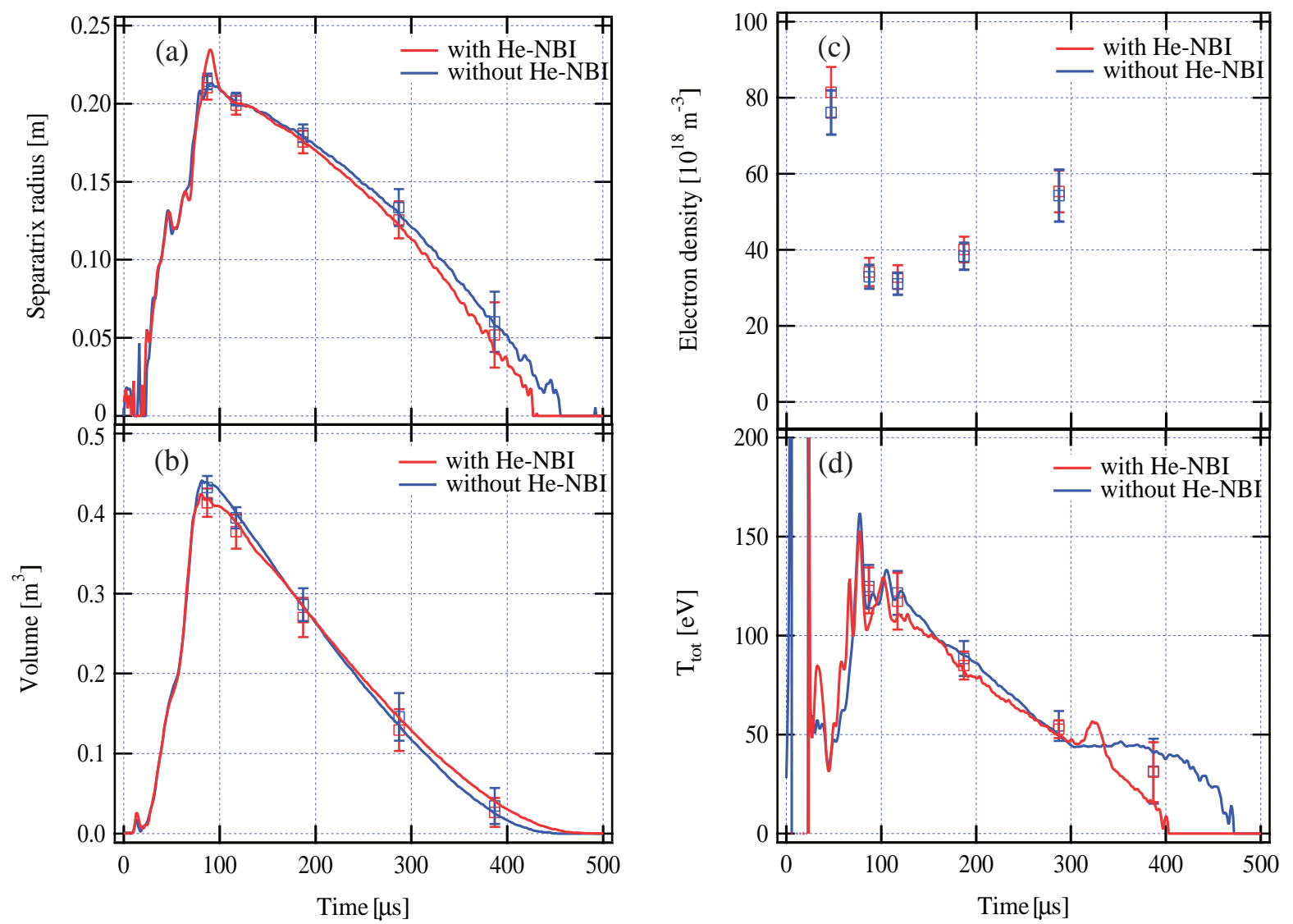

FIG.3 Same as Fig.2, except the beam is helium.

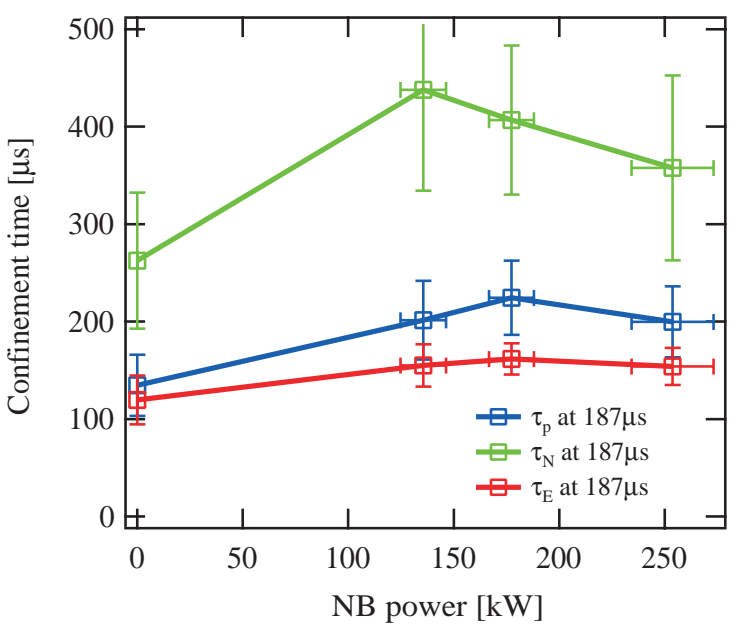

FIG.4 Dependence of confinement times on injected $N B$ power.

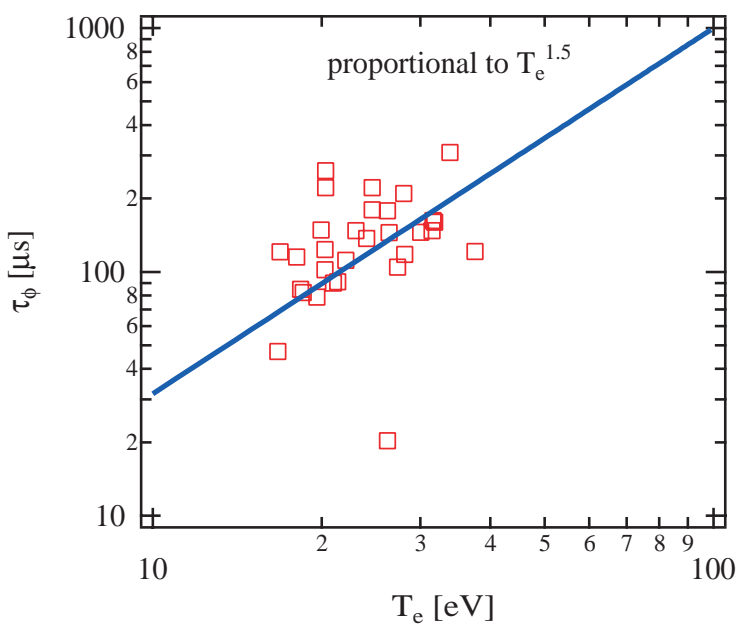

FIG.5 Dependence of $\tau_{\phi}$ on $T_{e}$. Dotted lines signifies $T_{e}^{3 / 2}$ dependence. 


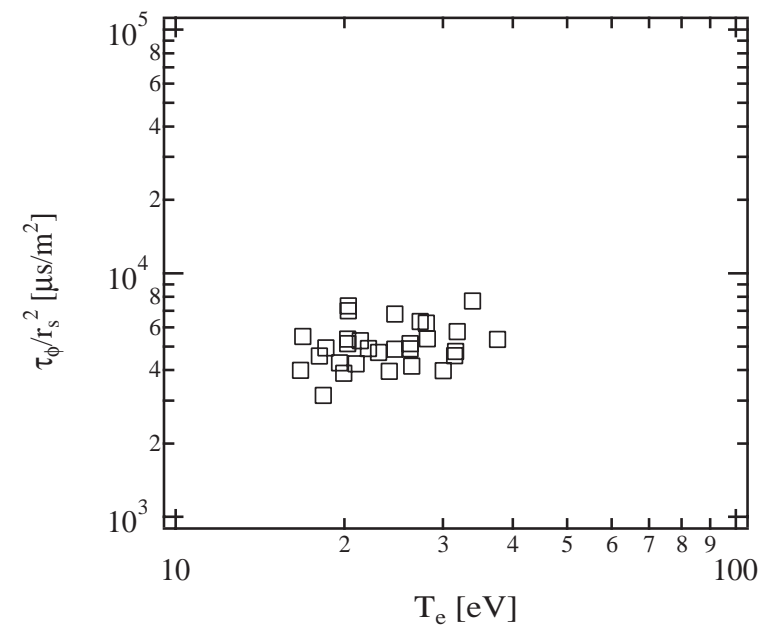

FIG.6 Dependence of $\tau_{\phi} / r_{s}^{2}$ on $T_{e}$.

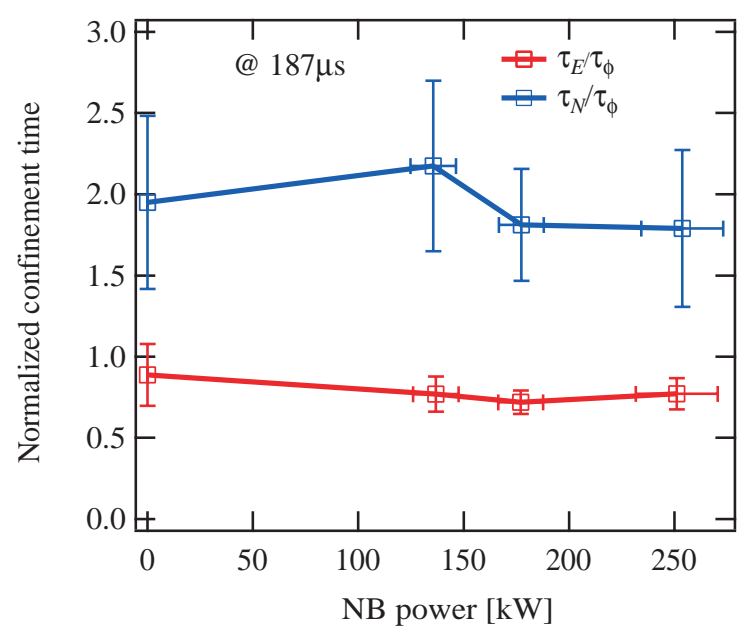

FIG.7 Dependence of $\tau_{N} / \tau_{\phi}$ and $\tau_{E} / \tau_{\phi}$ on NB power.

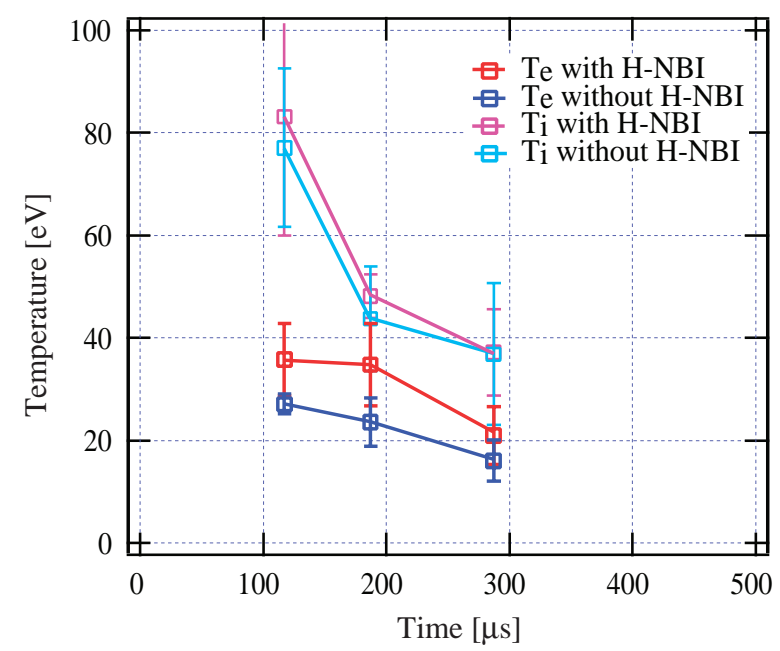

FIG.8 $T_{e}$ and $T_{i}$ with and withougt NB.

\section{Sustainment experiment}

At first, we tried to sustain translated FRC plasma with $n \cong 5 \times 10^{19} / \mathrm{m}^{3}$ and $T_{\text {tot }} \cong 100 \mathrm{eV}$ in the bias (confinement) field of about $50 \mathrm{mT}$ by the rotating magnetic field (RMF). No effect was observed probably due to the screening effect of RMF by ambient plasma. Then we switched to the experiment to produce and sustain the FRC plasma solely by the RMF. The RMF was applied to $\mathrm{H}_{2}$ gas filled to $0.03-1 \mathrm{~Pa}$ or to the after glow plasma originating from collapsed translated FRC plasma in the bias field of smaller than $1 m T$. In Fig.9, RF current fed to each antenna rod is shown (a) and the strength of the magnetic field at $r=0.01 \mathrm{~m}$ and $r=0.23 \mathrm{~m}$ (b). As a resonant tank circuit is adopted, the current is seen to build up until it decreases suddenly when the gas breaks down at $400 \mu \mathrm{s}$. The axial field is seen to be reversed at $600 \mu \mathrm{s}$, which signifies that the FRC plasma is produced. The RMF decreases gradually from $450 \mu$ s to $1150 \mu s$ because the electrical energy is provided from capacitors. 

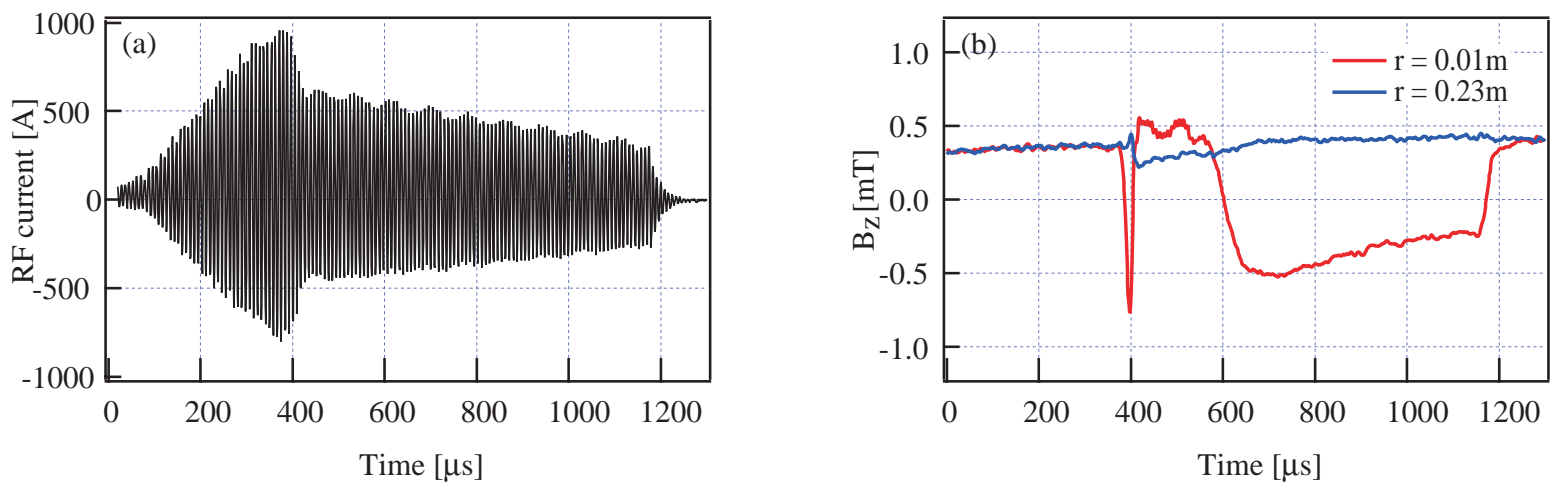

FIG.9 (a) RF current fed to each antenna rod. (b) Magnetic field at $r=0.01 \mathrm{~m}$ and $r=0.02 \mathrm{~m}$.

In Fig. 10, temporal development of the amplitude of the RMF $\left(B_{\theta}\right)$ at various radial location is shown. As the magnetic probes located at $r=25.4,22.4$ and $16.4 \mathrm{~cm}$ are located near one of antenna rods, they record larger RMF amplitude than other probes. When the plasma is formed at $130 \mu \mathrm{s}$, the amplitude becomes small deep inside the plasma. In Fig.11 (a), radial profile of the axial magnetic field strength at $500 \mu \mathrm{s}$ in the axial midplane measured by radial array of search coils is shown. In Fig.11 (b), radial profile of the toroidal current which is sustaining the FRC is shown. Even though the RMF attenuates deep in the plasma, the current is seen to be driven. In Fig.12, change of $r_{s}$ with time is shown. Assuming radial equilibrium, $r_{s}$ is calculated by $r_{s}=\sqrt{2} R$ using the value of $R$ obtained from the magnetic field profile at each time. In Fig.13, dependence of the toroidal current $I_{\theta}$ on $r_{s}$ is shown for different bias field. Solid lines are dependence of $I_{\theta}$ on $r_{s}$ calculated for the rigid rotor profile. Data points (colored solid circles) for each bias field scatter below the solid lines. Although the inner radius of the vacuum chamber is $40 \mathrm{~cm}$, the value of $r_{s}$ is limited to $25 \mathrm{~cm}$, because antenna rods are located at $r=25 \mathrm{~cm}$. In Fig.14, dependence of the depth of field reversal $\Delta B_{Z}$ or the difference between the maximum and the minimum magnetic field strength on the amplitude of driving RMF $B_{R M F}$ for different drive frequency is shown. Each solid line signifies upper bound of the performance for each frequency. Higher efficiency, or deeper reversal with smaller $B_{R M F}$, is observed for higher frequency. The efficiency is extremely poor at $58 \mathrm{kHz}$, when the drive frequency is close to the ion gyro frequency.

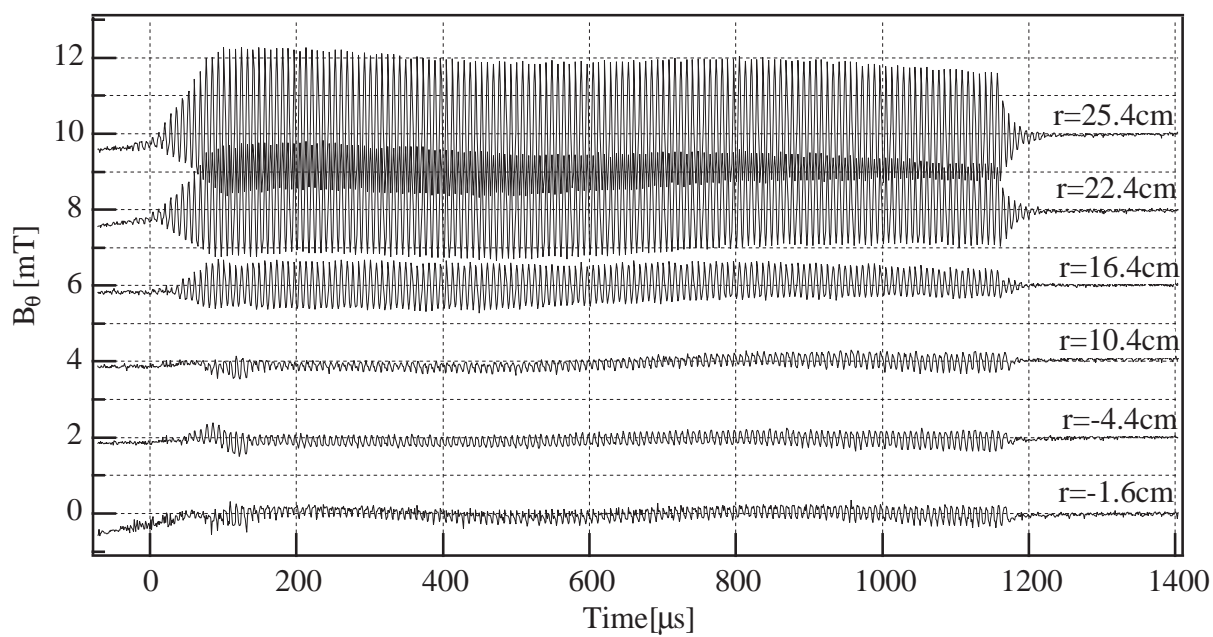

FIG.10 Temporal development of the RMF amplitude at various radial location. 

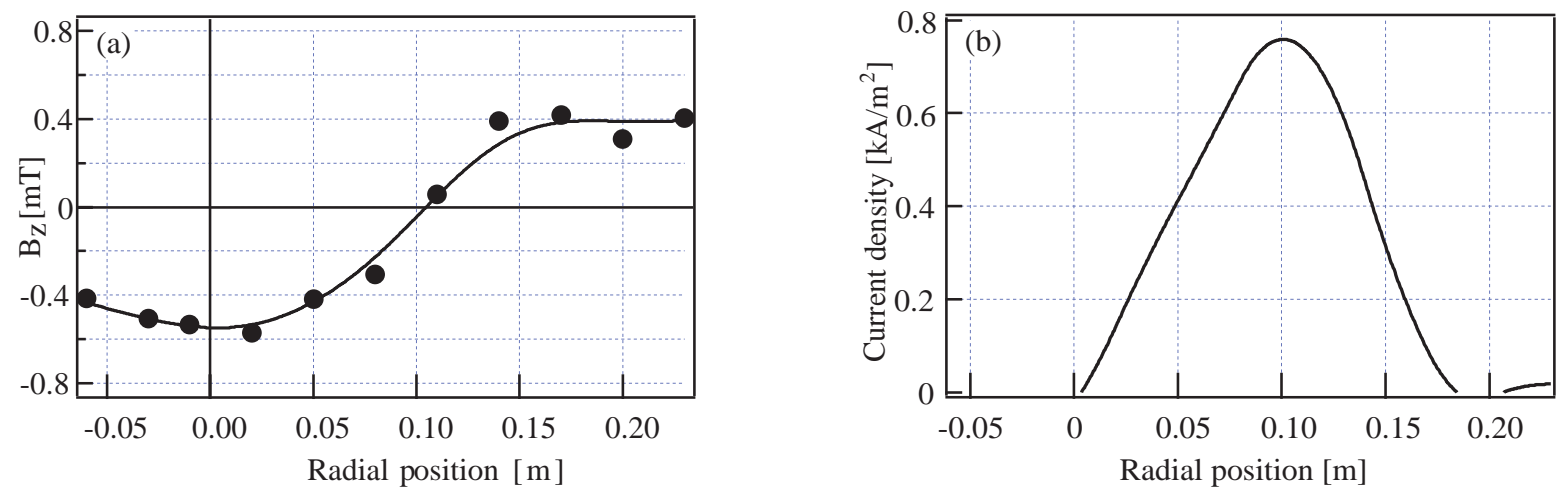

FIG.11 (a) Radial profile of the axial magnetic field. (b) Radial profile of the toroidal current.

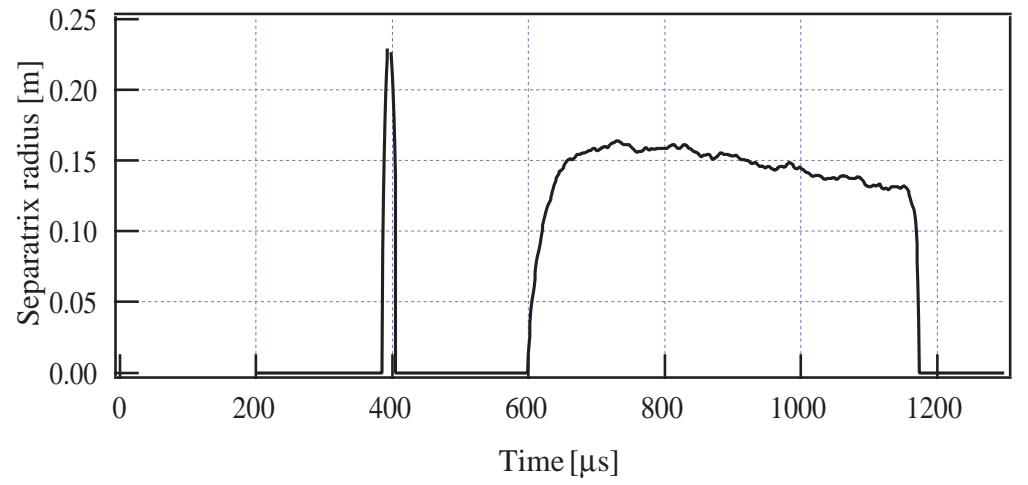

FIG.12 Change of $r_{s}$ with time.

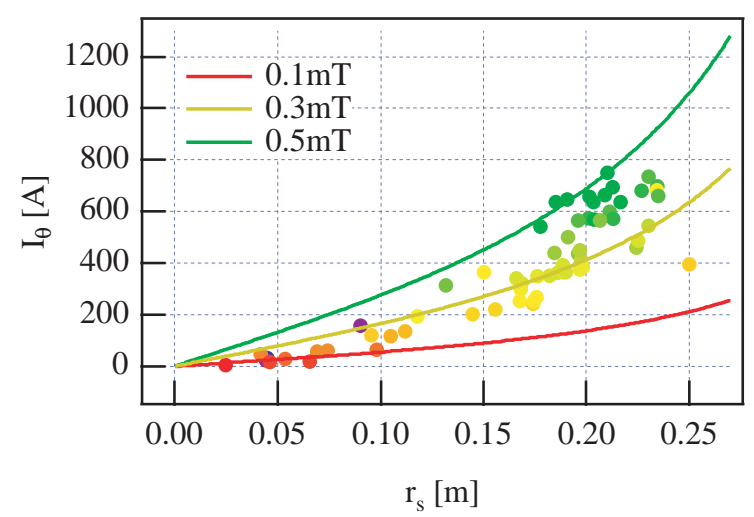

FIG.13 Dependence of the toroidal current $I_{\theta}$ on $r_{s}$ for different bias field. Solid lines are those for calculated for the rigid rotor profile

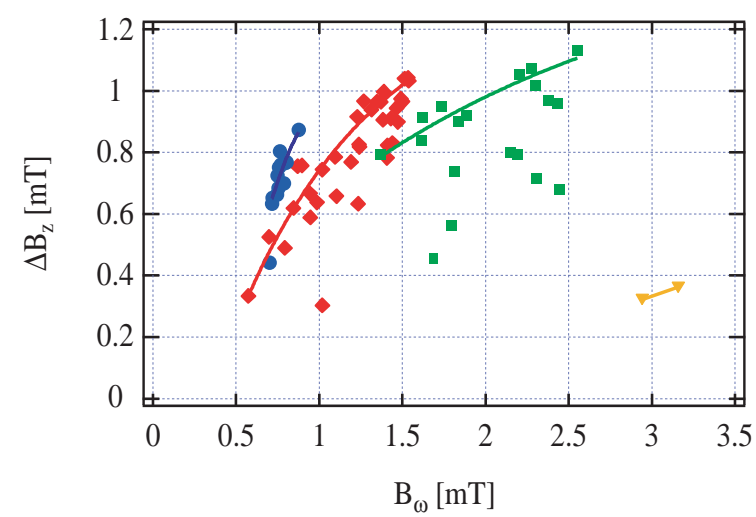

FIG.14 Dependence of the depth of field reversal on the driving $R M F$ amplitude 


\section{Conclusions}

Additional heating experiment on the translated FRC plasma with $n \cong 5 \times 10^{19} / \mathrm{m}^{3}$, $T \cong 10 \mathrm{keV}$ and $B_{w} \cong 50 \mathrm{mT}$ was accomplished with $10 \mathrm{keV}$ hydrogen neutral beam. Electron heating, but not ion heating, was observed in this experiment. So far, confinement times $\tau_{N}, \tau_{\phi}$ and $\tau_{E}$ were known to depend approximately on $R^{2} / \rho_{i}$ and $T_{e}$ dependence was small. In the present experiment in which $T_{e}$ and $T_{i}$ were controlled independently for the first time in the FRC plasma, it was found that confinement times do not depend on $T_{e}$ within experimental error.

The FRC plasma was produced and sustained by the RMF in the bias field of smaller than $1 m T$ in the metal chamber for the first time. Though the performance is low; $T_{e}$ and $n_{e}$ are less than $10 \mathrm{eV}$ and $5 \times 10^{17} / \mathrm{m}^{3}$ respectively and can be sustained in quartz vacuum chamber at present, future FRC plasma with higher temperature must be sustained in a metal chamber. Efficiency of the FRC plasma production was observed to be higher when the frequency of driving RMF is higher.

\section{References}

[1] TUSZEWSKI M., "FIELD REVERSED CONFIGURATIONS”, Nucl. Fusiion 28 (1988) 2033.

[2] OKADA, S., "Excitation and propagation of low frequency wave in a FRC plasma", Nucl. Fusion 43 (2003) 1140.

[3] YAMANAKA, K., et al., "Heating experiment of field-reversed configuration plasma by low-frequency magnetic pulse", 7 (2000) 2755.

[4] MOMOTA, H., et al., "THE D- ${ }^{3} \mathrm{He}$ FUELLED FIELD REVERSED CONFIGURATION REACTOR ARTEMIS-L", Plasma Physics and Controlled Nuclear Fusion Research 1992, (Proc. 14th Int. Conf. Wurzburg, 1992), IAEA, Vienna (1993) vol.3, 319-325.

[5] OAKDA, S., et al., "Experiments on additional heating of FRC plasmas", Nucl. Fusion 41 (2001) 625.

[6] ASAI, T., et al., "Experimental evidence of improved confinement in a high-beta field-reversed configuration plasma by neutral beam injection", Phys. Plasmas 7 (2000) 2294.

[7] HOFFMAN, A. L., "Flux buildup in field reversed configurations using rotating magnetic fields", Phys. Plasmas 5 (1998) 979.

[8] GUO, H. Y., "Formation and steady-state maintenance of field reversed configuration using rotating magnetic field current drive", Phys. Plasmas 9 (2002) 185. 